\title{
Jeden Sonntag Pfingsten? Evangelische Kirche - Vielfalt durch Migration
}

Michael Bünker

Churches have been deeply affected by migration since day one. This article recalls the experiences that the Protestant churches in Austria have had with migration and describes how migration currently affects them. It outlines the different models of coexistence between migrant congregations and those in the recipient society and traces migrant congregations' stages of development. It also presents and addresses the issues for churches and religious communities that research on migration has revealed, reflects upon practical experience, and suggests potential ways forward. Bünker's proposal for the coexistence of churches with different cultural and ethnic characteristics is to apply a model of church fellowship based on the "Leuenberg Agreement", as developed for the Protestant Churches in Europe.

Dr. theol. Michael Bünker was born in 1954. He has been the General Secretary of the "Community of Protestant Churches in Europe (CPCE)" since 2007 and the Bishop of the Protestant Church A.C. in Austria since 2008.

\section{Einleitung}

Jeden Sonntag Pfingsten? Auf der einen Seite klingt das nach Dauerstress und multikultureller Überforderung, auf der anderen aber steckt etwas Wahres drinnen, das helfen kann, die zunehmende Prägung durch Migration und ihre Folgen auf die evangelischen Kirchen zu deuten: ein vielsprachiges Durcheinander, oft und weithin Nebeneinander, und da und dort wachsendes Miteinander, das dadurch möglich wird, dass die Botschaft des Evangeliums unter dem Wirken des Geistes Gottes allen verständlich wird. Auf diesen Gedanken komme ich noch zurück.

\section{Zwei Erinnerungen}

Zuerst aber eine Erinnerung: Die Evangelische Kirche in Österreich zählt heute etwa 300000 Mitglieder. Es ist eine Kirche mit Migrationserfahrung, und zwar in doppelter Hinsicht: Zum einen durch die vom 16. Jahrhundert an bis zur Toleranz im Jahre 1781 vorherrschende Erfahrung der Vertreibung. Transmigration hat man diese Deportationen im 18. Jahrhundert euphemistisch genannt. Zehntau- 
sende Evangelische waren davon betroffen ${ }^{1}$. Ein letztes Nachglühen dieser unseligen Geschichte war die Vertreibung der Protestanten aus dem Tiroler Zillertal im Jahr 1834, die als letzte rein aus Glaubensgründen durchgeführte Deportation in Zentraleuropa gilt. Dieses Schicksal haben die Evangelischen hierzulande gemeinsam mit den Waldensern, den Hugenotten, den Täufern und vielen anderen, die um ihres Glaubens willen aus ihren Herkunftsländern verjagt worden sind.

Zugleich hat die Evangelische Kirche in Österreich Erfahrung mit der Aufnahme und Integration von Zuwandernden. In den Jahren 1944 und 1945 verdoppelte sich etwa in Oberösterreich die Bevölkerung insgesamt und damit auch die Zahl der Evangelischen durch Zuwanderung der Vertriebenen, Flüchtenden oder Umgesiedelten aus den südosteuropäischen Ländern, aus Rumänien und dem früheren Jugoslawien. ${ }^{2}$ Jahre bevor die Politik diese Zugewanderten aufgenommen hat, hat dies die Kirche getan. Der damalige Bischof Gerhard May hat es 1950 auf folgende kurze Formel gebracht:

Die evangelische Kirche kennt keinen Unterschied zwischen Österreichern und Flüchtlingen. Die evangelische Kirche macht keinen Unterschied zwischen „Displaced Persons" und Volksdeutschen. Da die erhoffte Rückkehr in die Heimat gar nicht möglich sein wird und nicht alle in Deutschland unterkommen oder nach Übersee auswandern können, werden sehr viele ihre neue Heimat in Österreich finden müssen. Die Kirche muss hier aktiv werden und dabei helfen. ${ }^{3}$

Freilich fügt Bischof May gleich hinzu, dass diese Grundsätze keineswegs überall und völlig durchgeführt wurden, und zwar weder von Seiten der Österreicher und Österreicherinnen noch von Seiten der Flüchtlinge. ${ }^{4}$ Ähnliche Erfahrungen in weit größerem Ausmaß und länger anhaltend - ich denke an die Aufnahme der Russlanddeutschen - hat die Evangelische Kirche in Deutschland gemacht. Migration, Flucht und Vertreibungen gehören also zur Geschichte der evangelischen Kirche und sind in deren kollektivem Gedächtnis fest verankert. ${ }^{5} \mathrm{Ob}$ diese Erinnerungen heute hilfreich sind?

Es ist interessant, dass sich schon damals, also in der Zeit zwischen 1945 und 1955, die Frage gestellt hat, ob es möglich sein wird, dass die Evangelischen der ansässigen, autochthonen Bevölkerung und die der Migranten und Migrantinnen miteinander eine Kirche bilden oder ob es, wie von manchen angestrebt, zur Bildung einer eigenen evangelischen Kirche der zugewanderten Vertriebenen und Geflüchteten in Österreich kommen würde. Dabei hatte man nicht nur das

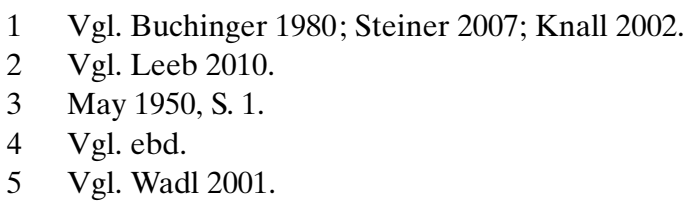


Bekenntnis und die Lehre gemeinsam, sondern auch die Sprache. Es wären also nur die aus heutiger Sicht gar nicht großen kulturellen Unterschiede gewesen, die womöglich zur Gründung einer eigenen Auslandskirche geführt hätten. Zum Glück ist es anders gekommen.

Schon damals stellte sich also die Herausforderung, „gemeinsam Kirche zu sein". Diese Herausforderung stellt sich durch die Migration unserer Tage wieder und nimmt ständig zu. Die migrationserfahrenen evangelischen Kirchen in Italien, allen voran die Waldenser, sprechen seit Jahren vom essere chiesa insieme, die Evangelische Kirche in Deutschland nennt es kurz und bündig „Gemeinsam evangelisch“. Gerade auch für die evangelischen Kirchen im deutschen Sprachraum ist das eine Herausforderung, denn seit Herder sind sie es gewohnt, Sprache, Kultur und Glaube als Einheit zu denken.

\section{Vom diakonischen zum ekklesiologischen Paradigma}

In der Einstellung der autochthonen evangelischen Kirchen zu den evangelischen Zugewanderten vollzog sich eine Entwicklung vom diakonischen zum ekklesiologischen Paradigma. In den 1960er-Jahren überwog die diakonische Motivation. Die Kirche wollte für „die Ausländer“ da sein, lange Zeit gab es die sogenannte „Gastarbeiterseelsorge“. Migrantische Gemeinden wurden als (hilfsbedürftige) Fremde wahrgenommen. Auch wenn dieses Paradigma heute überholt ist, wurde doch durch die gelebte Gastfreundschaft ein wichtiger Baustein hin zur Einwanderungsgesellschaft gelegt. Erst seit den 1990er-Jahren beginnt eine neue Entwicklung, die die migrantischen Gemeinden als ökumenische Partnerinnen versteht, der Wechsel vom diakonischen hin zum ekklesiologischen Paradigma ist vollzogen.

\section{Modelle des Miteinander}

Die konkrete Gestaltung des Miteinander lässt sich in verschiedenen Modellen beschreiben: Das „Parallel-Modell“: In den Räumlichkeiten einer in Österreich beheimateten evangelischen Gemeinde finden Gemeindeleben und Gottesdienste einer migrantischen Gemeinde statt. Simon beschreibt dieses nichtchristliche Nebeneinander von Geschwistern. ${ }^{6}$

So wichtig das Modell der (ökumenischen) Gastfreundschaft in der ersten Kontaktphase auch sein mag, es genügt nicht auf Dauer.

Das „Schwesterkirchen-Modell“: beide Gemeinden haben regelmäßig Kontakt und feiern - zumindest fallweise - gemeinsame mehrsprachige Gottes-

6 Vgl. Simon 2011, S. 95. 
dienste. Die Vertreter und Vertreterinnen der migrantischen Gemeinde sind in den Pfarrkonventen und kirchlichen Vertretungsorganen vertreten.

Das „Integrationsmodell“: Migrantische Gemeinden werden mit allen Rechten und Pflichten Gemeinden der ansässigen evangelischen Kirche. Dies geschieht meist durch Abkommen mit den Schwesterkirchen in den Herkunftsländern. Das ist der Weg, den auch die Evangelische Kirche in Österreich bevorzugt geht, auch wenn die „Einpassung“ der internationalen Gemeinden, wie sie manchmal auch genannt werden, in die oft schwer vermittelbaren Rahmenbedingungen nach dem hier geltenden inneren und äußeren Religionsrecht eine dauernde Herausforderung darstellt. Die Kooperation mit zivilgesellschaftlichen und politischen Kräften zeigt, dass dadurch die Kirchen einen wichtigen Beitrag zur angestrebten Integration der Zugewanderten leisten können.

\section{Verschiedene Ekklesiogenesen}

Im Miteinander ist zu berücksichtigen, dass sich die migrantischen Gemeinden und Kirchen sehr unterschiedlichen Entstehungsbedingungen, unterschiedlichen „Ekklesiogenesen“ verdanken. Benjamin Simon führt dies am Beispiel der afrikanischen Kirchen durch und stellt dabei drei Typen von „Ekklesiogenesen“ heraus ${ }^{7}$ : Zum ersten Typ gehören jene Kirchen, die sich bereits seit dem frühen 20. Jahrhundert von den Missionskirchen gelöst haben und nun Gemeinden in europäischen Ländern gründen. Als zweites sind die - so die Terminologie von Simon - „diasporalen“ Kirchen zu nennen, die ihren Ursprung nicht in Afrika, sondern im Residenzland haben. Ihre Mitglieder gehören nicht zur selben Herkunftskirche, sondern finden sich erst in der neuen Heimat zusammen, indem sie sich aus kleinen, zuerst meist auch ethnisch identifizierbaren und sprachlich homogenen Hauskreisen entwickeln. Mit der strukturellen Verfestigung geht oft auch die Namensgebung einher, diese Kirchen heißen zum Beispiel „All Christian Believers Fellowship“, „International Triumphant Church of Christ“" oder ähnlich. Die dritte Gruppe umfasst die Kirchen, die sich einer transkulturalen Ekklesiogenese verdanken. Das sind jene Kirchen, die ihren Ursprung in der Diaspora des Residenzlandes haben, aber bereits Gemeinden in den Herkunftsländern und anderswo gegründet haben. Als Beispiel nennt Simon die „Christian Church Outreach Mission“, die 1982 in Hamburg entstand und mittlerweile Gemeinden in Ghana, Togo, Nigeria, Washington D.C. und Amsterdam gegründet hat. Nicht wenige von ihnen verfolgen das Konzept einer „reverse mission“, d. h., sie betrachten Europa als Missionsgebiet und verstehen sich als gesandt, um die dem Christentum weitgehend entfremdeten Menschen im Residenzland für den Glauben (neu) zu gewinnen. ${ }^{8}$

7 Vgl. Simon 2011.

8 Vgl. Harfst 2011. 


\section{Dreiphasige Entwicklung}

Migrantische Gemeinden durchlaufen verschiedene Phasen der Entwicklung. Benjamin Simon hat dafür ein dreiphasiges Entwicklungsmodell vorgeschlagen. ${ }^{9}$ In der ersten Phase sind die Migrationskirchen vorwiegend mit inneren Findungsprozessen beschäftigt. Sie sind monoethnisch und monokulturell zusammengesetzt und unterhalten so gut wie keine ökumenischen Kontakte und entfalten keine (missionarische) Wirkung nach außen. Simon nennt das die „Phase der Seklusion“. In einer zweiten Phase („Phase der Öffnung“) führen die Migrationskirchen eine europäische Sprache als Gottesdienstsprache ein und öffnen sich für Mitglieder verschiedener ethnischer Herkunft. Sie werden vielsprachig und multikulturell und beginnen mit aktiver Missionsarbeit. In der dritten Phase („Phase der Interkulturation“) haben Europäer und Europäerinnen Zugang zur migrantischen Gemeinde gefunden und in ihr auch leitende Funktionen übernommen. Es kommt zu einem regen gegenseitigen Austausch im Sinn einer „Interkulturalität ${ }^{\star 10}$, die ökumenischen Verbindungen sind gefestigt. Die Beachtung dieser in Phasen stattfindenden Entwicklung, die allerdings nicht linear verlaufen muss, kann den Kirchen des Residenzlandes helfen, ihre Beziehungen zu den Migrationskirchen - in Wahrnehmung des jeweiligen Identitätsbewusstseins und sensibel gegenüber dem „Prozess des ständigen Balancierens“"11, den die Migrationskirchen zu bewältigen haben - zu gestalten.

\section{Modell: Kirchengemeinschaft}

Die Migrationskirchen stellen die ansässigen Kirchen vor neue Herausforderungen. Klassische Wahrnehmungsmuster und konfessionelle Zuordnungen werden infrage gestellt. Dabei geht es nicht um „Integration“ in dem Sinn, dass sich die Migrationskirchen ein- und anzupassen hätten, sondern um ein gegenseitiges Geben und Nehmen auf der Grundlage des Respekts und der Anerkennung. In diesem Prozess kommt es zu Veränderungen auf beiden Seiten, das ökumenische Miteinander verlangt nach Neuanpassung, nach einer Rekonfiguration.

Dafür bietet sich aus evangelischer Sicht das Leitbild der „Einheit in versöhnter Verschiedenheit" an. Dieses Leitbild wurde im Rahmen des Lutherischen Weltbundes in den 1970er-Jahren für die weltweite communio der lutherischen Kirchen entwickelt. Es ist geeignet, das Miteinander von Kirchen als Kirchengemeinschaft zu beschreiben. Für die evangelischen Kirchen in Europa ist Kir-

9 Vgl. Simon 2011, S. 92-94.

10 Zu diesem Begriff: vgl. ebd., S. 94.

11 Krappmann ${ }^{9} 2000$, S. $32 \mathrm{f}$. 
chengemeinschaft auf der Grundlage der Leuenberger Konkordie (1973) $)^{12}$ verwirklicht worden, die seit den 1990er-Jahren häufig als solche „Einheit in versöhnter Verschiedenheit" beschrieben wird. Die in diesem ökumenischen Einheitsmodell verwirklichte Kirchengemeinschaft besteht aus Kanzel- und Abendmahlsgemeinschaft, aus der gegenseitigen Anerkennung der Ämter und der möglichst großen Gemeinsamkeit in Zeugnis und Dienst in der Welt. Dieses Modell, das im Bereich der europäischen evangelischen Kirchen umgesetzt wurde, kann nun auch mutatis mutandis für das Verhältnis zu den migrantischen Gemeinden und Kirchen fruchtbar gemacht werden. Es geht dabei nicht - wie die Leuenberger Konkordie selbst feststellt - um eine „Vereinheitlichung, die die lebendige Vielfalt der Verkündigungsweisen, des gottesdienstlichen Lebens, der kirchlichen Ordnung und der diakonischen wie gesellschaftlichen Tätigkeit beeinträchtigt". Eine solche vielfaltsblinde Einheit würde direkt „, [...] dem Wesen der mit dieser Erklärung eingegangenen Kirchengemeinschaft widersprechen“ (Leuenberger Konkordie, Artikel 45). „Einheit in versöhnter Verschiedenheit“ könnte man auch als Einheit in Identität und Differenz bzw. als Einheit von Identität und Differenz bezeichnen. Gemeinsam Kirche sein nach dem Modell der „Einheit in versöhnter Verschiedenheit“ stellt die jeweilige Identität nicht infrage und bietet gleichzeitig für den Prozess des zusammen Wachsens und des Zusammenwachsens einen Deutungsrahmen in hoher ekklesiologischer Reflexivität.

Die Evangelische Kirche in Österreich ist bestrebt, die migrantischen Gemeinden ${ }^{13}$ in die Kirche zu integrieren, ihnen denselben (rechtlichen) Status zuzuerkennen wie den Gemeinden der ansässigen Kirche und ihnen dabei selbstverständlich ihre kulturelle Eigenart zu lassen. Miteinander machen die Evangelischen Kirchen sichtbar, wie diese Einheit in Verschiedenheit gewachsen ist und heute lebt. So sind sie selbst offen für Vielfalt und Pluralität und setzen das im Miteinander mit ihren zugewanderten Mitgliedern um. Dieses Miteinander kann unterschiedlich gestaltet sein. Es kann zur Gründung internationaler Gemeinden führen, die die gleichen Rechte und Pflichten haben, wie die eingesessenen Gemeinden der jeweiligen Kirche. Es kann zur Aufnahme der Zugewanderten in den bestehenden Gemeinden führen, was meist zur Folge hat, dass die Gottesdienste und das Gemeindeleben für alle vielsprachig und multikulturell werden. In jedem Fall bedeutet es, dass die zugewanderten Christinnen und Christen nicht bloß „Gäste“ sind, denen ein vorübergehendes Gastrecht eingeräumt wird. Die Grundintention ist in jedem Fall, sichtbar zu machen, was es heißt, gemeinsam evangelisch zu sein. Die gegenseitige Bereicherung durch neue Erfahrungen und Ausdrucksformen des Glaubens ist für alle Beteiligten ein Lernprozess, der die Verantwortung füreinander und das selbstverständliche Miteinander einschließt.

12 Der Text wurde dreisprachig neu aufgelegt: Bünker / Friedrich 2013.

13 Vgl. Evangelische Kirche A. und H.B. in Österreich und Evangelisch-methodistische Kirche 2015. 
Das hat zur Folge, dass sich die vertrauten ökumenischen Konstellationen verändern. Es bedeutet weiters, sich von der Konzeption der diakonischen Hilfsbeziehung, in der die Migrationskirchen als bloße Empfängerinnen gesehen und damit zu Objekten der Zuwendung werden, zu verabschieden. Gemeinsam Kirche zu sein führt dazu, die geistlichen Gaben in ökumenischer Weise im Sinne des „ecumenical resource sharing“ zu teilen. Vielsprachigkeit und Interkulturalität sind in dieser Sicht nicht in erster Linie als Gefahr zu sehen, sondern als eine Bereicherung durch Vielfalt. Dies gilt auch für das gemeindliche Leben und die Vielfalt des gottesdienstlichen Lebens. Die migrantischen Gemeinden machen etwas vom weltumspannenden Leib Christi sichtbar und sie ermöglichen es auch der Kirche in der Residenzgesellschaft, die weltumfassende Kirche erlebbar und erfahrbar zu machen. Mit ihnen gemeinsam wird etwas von der Katholizität der Evangelischen Kirche sichtbar. So kann Migrations- und Residenzkirchen eine „Einheit in versöhnter Verschiedenheit“" eröffnet werden, in der sie miteinander ihrem Auftrag nachkommen und gemeinsam die eine Kirche Jesu Christi sichtbar machen. Dieser Beitrag der Kirchen zum integrativen Zusammenleben und interkulturellen Lernen ist auch ein Beitrag zum gesellschaftlichen Frieden in einer zunehmend multiethnisch geprägten Gesellschaft.

\section{Literaturverzeichnis}

Buchinger, Erich: Die „Landler“ in Siebenbürgen. München 1980.

Bünker, Michael / Friedrich, Martin (Hg.): Konkordie reformatorischer Kirchen in Europa (Leuenberger Konkordie). Leipzig 2013.

Evangelische Kirche A. und H.B. in Österreich und Evangelisch-methodistische Kirche (Hg.): Evangelische Internationale Gemeinden in Österreich. Wien 2015.

Harfst, Ursula: „Reverse Mission“, in: Evangelisches Missionswerk in Deutschland (Hg.): Zusammen wachsen. Weltweite Ökumene in Deutschland gestalten. Hamburg 2011, S. 29-40.

Knall, Dieter: Aus der Heimat gedrängt. Letzte Zwangsumsiedlungen steirischer Protestanten nach Siebenbürgen unter Maria Theresia. Graz 2002.

Krappmann, Lothar: Soziologische Dimensionen der Identität. Stuttgart ${ }^{9} 2000$.

Leeb, Rudolf: „Die Heimatvertriebenen und Flüchtlinge nach 1945 und die evangelische Kirche in Österreich: Auswirkungen der Migration auf eine ,Diasporakirche““, in: Rieske, Uwe (Hg.): Migration und Konfession. Konfessionelle Identitäten in der Flüchtlingsbewegung nach 1945 (= Die Lutherische Kirche Band 27). Gütersloh 2010, S. 167-201.

May, Gerhard: „Die Verantwortung der evangelischen Kirche für die Flüchtlinge, Neuland“, in: Wochenblatt der Donauschwaben (3/5,4) 1950.

Simon, Benjamin: „Identität und Ökumene. Das Beispiel von Christen afrikanischer Herkunft in der europäischen Diaspora“, in: Evangelisches Missionswerk in Deutschland (Hg.): Zusammen wachsen. Weltweite Ökumene in Deutschland gestalten. Hamburg 2011, S. 86-98. 
Steiner, Stephan: Reisen ohne Wiederkehr. Die Deportation von Protestanten aus Kärnten 1734-1736. Wien / München 2007.

Wadl, Wilhelm (Hg.): Glaubwürdig bleiben. 500 Jahre protestantisches Abenteuer, Katalog und wissenschaftlicher Begleitband zur Kärntner Landesausstellung. Friesach / Klagenfurt 2011. 